\title{
Les effets du numérique sur l'éducation, une note de lecture sur l'ouvrage de Georges-Louis Baron et Christian Depover
}

\author{
The effects of digital technology on education, a reading note on \\ the book by Georges-Louis Baron and Christian Depover
}

\section{Los efectos de la tecnología digital en la educación, notas de lectura sobre el libro de Georges-Louis Baron y Christian Depover}

\author{
Hervé Daguet, Le Centre interdisciplinaire de recherche normand en éducation \\ et formation (Cirnef Ea 7454) \\ Université de Rouen Normandie, France \\ herve.daguet@univ-rouen.fr
}

\begin{abstract}
RÉSUMÉ
Georges-Louis Baron est professeur émérite en Sciences de l'éducation à l'université de Paris et Christian Depover est professeur à l'université de Mons. Dans l'ouvrage paru en juillet 2019 dont ils sont les coordinateurs, ils traitent de la question des effets du numérique sur l'éducation. De par la richesse des références auxquelles ils font appel, ils montrent en quoi cette question d'actualité n'est en fait qu'une continuité de celles que les chercheurs, mais aussi les praticiens ou encore les prescripteurs, se posent depuis bien des années. Parmi les nombreux points forts de cet ouvrage, nous notons qu'il s'inscrit dans une tradition universitaire qui cherche avant tout à présenter une vision distanciée des effets du numérique sur l'éducation. La qualité de cet ouvrage porte également dans les analyses sur les diversités qui sont mises en avant. Ce livre est à recommander à tous ceux qui souhaiteraient s'informer sur les effets réels du numérique sur l'éducation et la formation.
\end{abstract}

Mots-clés : éducation, numérique, technologie 
Georges-Louis Baron is Professor Emeritus in Educational Sciences at the University of Paris and Christian Depover is Professor at the University of Mons. In the book published in July 2019, of which they are the coordinators, they deal with digital technology's effects on education. The wealth of references they use shows how this topical issue is only a continuation of what researchers, practitioners, and even prescribers have been asking themselves for many years. Among the many strengths of this book we note that it is part of an academic tradition that seeks above all to present a distant vision of the effects of digital technology on education. The quality of this work also lies in the analyses of the diversities that are put forward. This book is to be recommended to learn about the real effects of digital technology on education and training.

Keywords: education, digital learning, technology

\section{RESUMEN}

Georges-Louis Baron es profesor emérito de ciencias de la educación en la Universidad de París y Christian Depover es profesor de la Universidad de Mons. En el libro publicado en julio de 2019, del que son coordinadores, se trata la cuestión de los efectos de la tecnología digital en la educación. A través de la riqueza de referencias que utilizan, muestran cómo este tema de actualidad es de hecho una continuación de los temas de interés de investigadores, pero también de profesionales e incluso de prescriptores, desde hace muchos años. Entre los muchos puntos fuertes de este libro, observamos que forma parte de una tradición académica que busca principalmente presentar una visión distante de los efectos de la tecnología digital en la educación. La calidad de esta obra también radica en los análisis de las diversidades que se plantean. Este libro es recomendable para todos aquellos que quieran conocer los efectos reales de la tecnología digital en la educación y en la formación.

Palabras clave: educación, aprendizaje digital, tecnología

\section{Référence :}

Baron, G.-L. et Depover, C. (dir.). (2019). Les effets du numérique sur l'éducation : Regards sur une saga contemporaine. Villeneuve d'Ascq, France : Presses Universitaires du Septentrion.

Georges-Louis Baron est professeur émérite en Sciences de l'éducation à l'université de Paris (laboratoire EDA) et Christian Depover est professeur à l'université de Mons. Dans l'ouvrage paru en juillet 2019 aux Presses Universitaires du Septentrion (Université de Lille - Villeneuve d'Ascq), dont ils sont les coordinateurs, ils traitent de la question des effets du numérique sur l'éducation. De par la richesse des références auxquelles ils font appel, ils montrent en quoi cette question d'actualité n'est en fait qu'une continuité de celles que les chercheurs, mais aussi les praticiens ou encore les prescripteurs, se posent depuis bien des années, à une époque où l'on ne parlait pas encore d'ordinateur et d'Internet. 
Dans un premier temps nous présenterons une synthèse des contenus des chapitres puis, s'agissant également d'une lecture critique, nous dégagerons les points forts de ce livre mais également nous proposerons quelques éléments qui pourraient être soumis à discussion.

Le livre se divise en deux parties; la première est écrite par les deux éditeurs, Georges-Louis Baron et Christian Depover. Après un rapide préambule dans lequel ils introduisent l'ouvrage, les auteurs proposent 5 chapitres présentant 5 thématiques distinctes. Cette première partie est nommée « Cadre d'analyse ».

Ils commencent tout d'abord par s'interroger sur les effets du numérique d'un point de vue systémique en questionnant les effets sur le système éducatif. En effet, comme l'indiquent les auteurs, nombre de discours sur l'innovation et les technologies ont longtemps été porteurs d'espoir. Cependant ils ont également bien souvent permis de mettre en évidence des résistances humaines ou encore technologiques, qui de fait, limitent les effets escomptés. La compatibilité entre école et TIC est même questionnée dans ce chapitre.

La seconde thématique interrogée est celle des curricula. C'est une question récurrente dont les systèmes éducatifs se sont emparés de façon bien diverse. En dehors des questions classiques liées à l'éducation au numérique et avec le numérique les auteurs montrent comment les systèmes éducatifs intègrent ou non l'éducation aux médias à leurs curricula respectifs.

Dans le chapitre suivant, les auteurs questionnent le lien entre usage et compétence. Ce chapitre fait référence à de nombreux travaux qui sont bien antérieurs au numérique et montre comment, à l'époque des technologies éducatives, la question de l'acquisition des compétences était investiguée et mesurée.

Le chapitre 4 porte quant à lui sur le lien entre apprentissage ou méthode d'apprentissage et numérique. Un peu plus court que les précédents, il permet de faire le point sur l'influence des artefacts sur les méthodes pédagogiques ou les didactiques mises en œuvre par les enseignants, comme celles liées au constructivisme ou encore plus récemment au rôle que peut tenir l'enseignant participant à un MOOC.

Enfin dans le dernier chapitre, écrit par les co-éditeurs de l'ouvrage, ils s'intéressent aux effets du numérique sur le fonctionnement cognitif et social. Ils montrent ainsi que bien avant les recherches en neurosciences des travaux ont déjà analysé les effets des logiciels puis des hypermédias sur les capacités d'apprentissage des apprenants. Une autre perspective présentée ici est celle des liens qui peuvent exister entre ces usages et l'influence de nos comportements sociaux. Un point est également fait sur les digital natives et leurs rapports aux apprentissages via le numérique.

La seconde partie de l'ouvrage intitulée "Synthèses thématiques » est constituée de 8 chapitres écrits par 10 auteurs. Dans ces derniers sont proposées des études de cas permettant de mettre en avant les effets du numérique en éducation auprès de différents publics, enfants, adultes... ou encore dans différents contextes géopolitiques.

Les chapitres 6 et 7 respectivement écrits par Geoffrey Sockett et Thérèse Laferrière traitent de la question des effets du numérique sur les apprentissages. Le premier auteur, après avoir rappelé les distinctions entre informel, formel et non formel, se réfère notamment à des définitions du Conseil de l'Europe pour indiquer en quoi ces activités découlant de la vie quotidienne peuvent contribuer à ce que chaque individu construise ses apprentissages. II propose ensuite des perspectives; sachant en effet que $80 \%$ de nos futurs apprentissages seront liés à la sphère de l'informel, le numérique ne pourrait que prendre une place importante dans l'acquisition de ces derniers. En ce qui concerne maintenant Laferrière, cette dernière rappelle que les apprentissages collaboratifs ont été questionnés par les praticiens et les chercheurs bien avant la venue de l'ordinateur, des tablettes ou bien encore de l'Internet. En effet, Freinet ou bien encore les néopiagétiens comme Perret Clermont le préconisaient sans forcément avoir recours à la dimension 
technologique. L'auteure met aussi en lien dans ce chapitre le travail collaboratif et le numérique en rappelant l'importance des affordances. Intrinsèquement les technologies numériques ne sont pas à l'origine des effets sur les apprentissages, mais ces dernières seraient par contre directement en lien avec les usages, les pédagogies ou les didactiques. L'affordance permettrait alors pour un artefact de suggérer les actions possibles à l'apprenant. Enfin, même si l'auteure indique que l'apprentissage collaboratif pourrait alors être une voie intéressante, elle indique que celle de la personnalisation des apprentissages le serait probablement davantage.

Dans le chapitre suivant Viviane Glikman revient sur 30 ans d'usages du numérique dans le champ de la formation des adultes. Elle remet en perspective le développement des technologies via ces nouveaux médias, comme par exemple en France le cas de la télématique avec le Minitel, et le souhait de mettre en place des dispositifs pour former les adultes. C'est toutefois dans l'euphorie de l'arrivée de l'Internet que se sont développées massivement les formations à distance pour les adultes. Certains, après y avoir vu " un nouvel âge pour l'humanité ", y ont également vu " un nouvel âge pour la formation », qui permettrait de réduire les inégalités. Toutefois ces nouveaux modes d'apprentissage ont amené de nouvelles difficultés pour l'apprenant adulte qui sont liées à la médiatisation des contenus de formation ou encore à la maitrise des fonctionnements technologiques. Après une rapide présentation des apports des MOOC dans la formation des adultes, elle confirme que la dimension numérique est plus que tout devenue incontournable dans la mise en place des dispositifs de formation liés à ces publics.

Les chapitres 9 et 10 présentent ensuite des études de cas liées aux pays du Sud. Tout d'abord, Jacques Wallet et Pierre-Jean Loiret remettent en question une vision qui présenterait le développement des usages du numérique dans les pays du Sud comme pessimiste et très stéréotypé. Comme on peut également le voir dans le chapitre suivant, en Afrique, le développement du numérique et du numérique en éducation ne s'opère pas comme dans les pays du Nord mais semble être fortement lié à celui de la téléphonie mobile. De grandes collaborations de développement sont aussi menées entre Nord et Sud, comme l'initiative francophone pour la formation à distance des maitres (IFADEM). D'après les auteurs, il faudrait toutefois relativiser les effets constatés sur les bonnes pratiques de formations car comme toujours, rien ne permet de les dupliquer à grande échelle. Erwan Le Quentrec évoque ensuite le cas de l'apprentissage mobile dans le même contexte, dans les pays du Sud. En se référant à des données récentes, il confirme le taux important de pénétration du téléphone portable et du smartphone en Afrique. Les usages du numérique via des supports mobiles permettraient par exemple, de façon non exhaustive, de pallier le manque de ressources éducatives papier, d'éduquer les enfants dans les zones de guerre ou encore de proposer des formations professionnelles pour les apprenants du secteur informel.

Dans le chapitre suivant, Béatrice Drot-Delange présente le cas de l'éducation au numérique. Celle-ci a pour principal objet la transmission d'une culture numérique. Cette culture, comme l'auteure l'indique, est liée à une demande sociale, notamment dans le but de protéger les individus face à une accélération du développement des technologies numériques. Elle doit d'après elle être également considérée comme une éducation citoyenne. Cette culture s'est développée parallèlement à la culture informatique qui, elle, est liée à l'algorithmie ou encore à la programmation et son avatar actuel, le codage. À l'heure d'Internet dans les processus de développement des citoyens, il semblerait donc vain, d'après l'auteure, de chercher à séparer éducation au numérique et éducation à l'informatique.

Dans le chapitre 12 Éric Bruillard revient sur les origines des réflexions qui ont été menées afin de faire le lien entre apprentissage du numérique et évaluation ou certification des compétences. En France c'est dès 2000 que l'on trouve dans le Bulletin Officiel de l'Éducation Nationale ce désir de mettre en place ce qui deviendra le Brevet Informatique et Internet (B2I) au primaire puis au secondaire, actuellement la plateforme d'évaluation PIX. II montre à la fois que ce n'est pas uniquement un projet français mais qu'il s'inscrit dans une dynamique européenne comme par exemple le JRC's Learning and Skills project. L'auteur présente également dans ce chapitre les outils, comme GiBii, qui en France ont permis de gérer 
l'évaluation de ces compétences au sein du B2I. Enfin Bruillard voit dans les usages des blockchain ou encore des open badge en éducation, la poursuite des travaux menés auprès des élèves permettant l'évaluation de leurs compétences numériques.

Enfin le treizième et dernier chapitre écrit par Simon Collin et Périne Brotcorne utilise l'approche sociocritique comme grille de lecture pour analyser les effets du numérique en éducation. Les auteurs rappellent tout d'abord que bien souvent c'est sous l'angle de l'efficacité ou de la recherche d'amélioration qu'ont été appréhendées les recherches sur le numérique en milieu scolaire. L'approche sociocritique est d'abord une approche sociologique; elle permet notamment d'analyser tout ce qui précède aux usages du numérique chez les acteurs du système éducatif mais également de les analyser au sein de la pluralité des acteurs et surtout de leurs contextes de vie. À cette dimension sociologique on ajoute une dimension critique qui permet de dépasser la phase descriptive pour mieux appréhender les jeux de pouvoirs entre les différents acteurs sociaux. Ainsi même si les auteurs remettent en cause un couplage réducteur " effetefficacité " dans l'analyse du numérique à l'école, ils précisent que l'approche sociocritique permet de mettre au jour des effets non immédiats, donc dans le prolongement des usages en classe. Enfin cette approche a également pour objet d'analyser les fractures et diverses inégalités liées au numérique.

De façon plus globale, parmi les nombreux points forts de cet ouvrage, nous notons qu'il s'inscrit dans une tradition universitaire qui cherche avant tout à présenter une vision distanciée des effets du numérique sur l'éducation.

En effet, dans le cadre de la recherche en éducation et formation, ce sujet est très sensible et il eut été aisé d'adopter une posture partisane. La proximité des acteurs avec les technologies conduit parfois à ce qu'ils se centrent essentiellement, tels des technophobes, sur les effets négatifs de ces dernières sur l'éducation, soit au contraire, chez les technolâtres, sur les effets positifs du numérique. Ainsi, dans cet ouvrage chaque point de vue est analysé avec circonspection et surtout richement argumenté. De façon générale les auteurs se montrent assez prudents; ainsi ils confirment tous que des effets observés dans un cadre expérimental ne le seraient probablement pas dans le cadre d'une généralisation. Dans l'approche sociocritique, on entrevoit également qu'il serait vain, pour analyser des effets du numérique en éducation, de se focaliser sur la sphère de l'école car les usages du numérique sont aussi effectués en famille dans le cadre d'apprentissages formels et informels mais pas uniquement.

La qualité de cet ouvrage porte également dans les analyses sur les diversités qui sont mises en avant :

- La première concerne la diversité des approches théoriques convoquées. Même si ces approches font globalement référence aux Sciences de l'éducation et de la formation, elles sont en fonction des auteurs, diverses et complémentaires: approches sociologiques, psychologiques, pédagogiques ou didactiques, systémiques.

- La diversité vient également des contextes investigués. Ils sont majoritairement issus d'observations faites dans les pays du Nord mais deux chapitres sont explicitement dédiés à ceux du Sud. On retrouve aussi dans les différentes parties, çà et là des exemples tirés d'usages dans les pays du Sud.

- Enfin la diversité porte également sur les publics investigués. Cet ouvrage ne se focalise pas sur les usages numériques des enseignants mais se centre également sur les apprenants (enfants et adultes) et, même si c'est beaucoup moins prégnant, sur d'autres acteurs comme les acteurs institutionnels de la sphère éducative ou encore tout simplement les parents. 
Enfin la quarantaine de pages qui constitue la bibliographie atteste également de la qualité des textes de référence qui ont été choisis par les auteurs mais également de la richesse qu'ils ont pu apporter dans le cadre de l'argumentation.

II convient toutefois de formuler également quelques points qui auraient pu être améliorés. Le premier fait écho à la remarque précédente. En effet, on peut toutefois se demander si, d'une certaine façon, cet argument ne pourrait parfois pas être retourné. La plupart des auteurs, sans aucun doute dans le souci d'être le plus exhaustif possible, ont parfois eu tendance à multiplier les exemples alors qu'il aurait sans doute été préférable d'en réduire le nombre afin de les étudier plus en profondeur. Ces présentations encyclopédiques conduisent le lecteur à atteindre parfois la limite de la surcharge.

Toujours dans le domaine de l'argumentation, on aurait aimé dans certains chapitres avoir parfois des références récentes, mais nous le savons bien, le temps universitaire n'est malheureusement pas toujours en adéquation avec l'évolution rapide des usages du numérique en éducation et en formation.

Dans le même ordre d'idée, une des forces de l'ouvrage est de présenter à la fois des résultats issus de la recherche universitaire mais également d'organismes institutionnels comme l'OCDE avec PISA. On voit d'ailleurs chez Bruillard une volonté de les distinguer; il a ainsi consacré la fin de son article à recenser des sources issues de sites et de documents officiels. Cependant dans les chapitres ces sources bien souvent s'entrecroisent. Bien évidemment le lecteur expert sera à même de faire la part des choses entre ces différents types de documents, d'analyser les enjeux politiques, économiques et sociaux intrinsèquement liés à ces publications institutionnelles, mais quid du non expert?

La dernière critique qui pourrait apparaitre aisée à formuler et valide quel que soit l'ouvrage coordonné autour d'une thématique est le fait que l'on retrouve parfois des éléments redondants entre les différents chapitres. On se doit donc de minimiser cette critique en argumentant que ces éléments redondants ne le sont jamais de façon contradictoire entre les chapitres mais surtout qu'ils le sont de façon différente au sein de contextes distincts.

Quoi qu'il en soit, ce livre est à recommander à tous ceux qui souhaiteraient s'informer sur les effets réels du numérique sur l'éducation et la formation. Les néophytes y trouveront des réponses à leurs interrogations et les experts, au-delà de la confirmation de leurs connaissances, y trouveront obligatoirement des éléments qui permettront de la compléter. 